\title{
Childbirth Educators' Role Regarding Pregnant Women and Cervical Cancer Prevention
}

Olufunmiso Oyetunde Asamu, $\mathrm{MPH}, \mathrm{PhD}$

Wellstar Kennestone Hospital, Marietta, Georgia, United States

Hadi Danawi, MPH, PhD

Walden University, Minneapolis, Minnesota, United States

iD https://orcid.org/oooo-0002-7288-4189

Contact: Hadi.Danawi@mail.waldenu.edu

\section{Abstract}

Childbirth educators play an important role in educating pregnant women regarding their health. They bridge the gap between pregnant women and healthcare providers. A paucity of information exists on the benefits that pregnant women derive from interactions with childbirth educators regarding early cervical cancer detection. The purpose of our cross-sectional study was to investigate the association among education during early pregnancy offered by the childbirth educators, access to childbirth educators, availability of childbirth educators in the United States and survival from cervical cancer. Secondary data from the Surveillance, Epidemiology, and End Results (SEER) on 520,153 women were analyzed using logistic regression. Childbirth education delivered by childbirth educators during early pregnancy was associated with significantly fewer cervical cancer deaths (odds ratio $=.917, C I=.896-.939, P=.000$ ). Women with late-stage diagnosis had an increased likelihood of dying when diagnosed with cervical cancer (odds ratio $=1.043, C I=1.042-1.044, P=$ .ooo). Childbirth educators are effective at increasing awareness of cervical cancer among women in the early stages of pregnancy; this can improve survival and lead to positive social change.

Keywords: cervical cancer, childbirth educators, human papilloma virus (HPV)

Date Submitted: May 19, 2020 | Date Published: November 8, 2021

Recommended Citation

Asamu, O. O., \& Danawi, H. (2021). Childbirth educators' role regarding pregnant women and cervical cancer prevention. Journal of Excellence in Nursing and Healthcare Practice, 3(1), 23-31. https://doi.org/10.5590/JENHP.2021.3.1.02

\section{Introduction}

Cervical cancer prevalence is a concern in the United States. Kervella et al. (2020) reported that human papillomavirus (HPV) is responsible for a significant portion of cervical cancer cases, and early detection and early treatment improve survival. Further, health education for pregnant women remains the only tool to reduce the incidence and prevalence of cervical cancer; childbirth educators assist pregnant women in making decisions regarding cervical cancer screening. Childbirth educators are healthcare professionals who offer health information to pregnant women during antenatal, natal, and postdelivery visits (Cutajar et al., 2020). 
Childbirth educators are trained to educate pregnant women on the need to register for antenatal care during pregnancy, delivery, and care for the babies and themselves after delivery. Childbirth educators are trained as either nurses or strictly as childbirth educators (Waller-Wise, 2011). Waller-Wise (2011) reported that childbirth educators are one of the main sources that expectant mothers depend on for important information to make informed decisions about their health during and after pregnancy and birth. Childbirth educators coached women in health education classes, and women expressed confidence that the teaching prepared them for pregnancy, birth, and postnatal events (Waller-Wise, 2011).

Childbirth educators' roles are minimal in hospital settings despite the level of knowledge they possess (Waller-Wise, 2011). Childbirth educators encourage pregnant women to receive adequate immunization during pregnancy and take their babies for immunization after delivery (Luce et al., 2016). According to DeebSossa and Kane (2017), in early 2000, the United States supported prenatal classes that undermined pregnant women's autonomy and that, until today, limit the power of women to acquire the knowledge necessary to adequately prepare for delivery.

\section{Background}

Globally, cervical cancer is the number two cause of malignant cancer in women after breast cancer (Zhang et al., 2020). The World Health Organization (WHO) declared cervical cancer a world health concern on November 17 , 2020 and mobilized 194 member countries to embark on prevention strategies to eliminate cervical cancer through health education (Zhang et al., 2020). Pregnant women and their partners need the information on health promotion during pregnancy, birth, and the postnatal period offered by the childbirth educators (Davis et al., 2019). Childbirth educators help pregnant women make good health decisions regarding birth and parenting (Deeb-Sossa \& Kane, 2017). For example, Zauderer (2009) argued that women are not allowed to acknowledge postpartum depression as a viable phenomenon, remaining silent, simply because women are expected to be joyful having a newborn. Childbirth educators can play a significant role in reshaping this attitude and recognizing postpartum depression as a medical phenomenon requiring medical attention.

Half of the world population is below 25 years of age, and $90 \%$ of them are living in low- and middle-income countries; the adolescent population accounts for $23 \%$ of the world's burden of diseases related to pregnancy and childbirth, with $11 \%$ of world births given by mothers between the ages of 15 and 19 years (Furry et al., 2019). Pregnant women with high-risk HPV results are associated with high HPV infection before pregnancy, often linked with early age of first teenage sexual intercourse (Suzuki et al., 2021). Carcinoma of the cervix is one health issue associated with women who have experienced multiple births and is preventable when women receive the health information that enables them to be tested for HPV infection early in their childbearing years (Sherris et al., 2001).

Research has shown that empowering the role of childbirth educators and other health-related teachers to give pregnant women and teens the necessary education on risk factors, how to avoid them, and good healthcare decision making reduced the incidence and prevalence of cervical cancer (Mamo \& Epstein, 2017).

Childbirth educators spend more time with pregnant women offering health education on habits that lead to safe delivery (Gower et al., 2016). Radecki Breitkopf et al. (2016) reported that socioeconomically disadvantaged women lack the knowledge of risk factors associated with cervical cancer development and prevention. Radecki Breitkopf et al. (2016) reported that childbirth educators offered help at birth to teach women the importance of the HPV vaccine for their male and female offspring. Pregnancy is a long and sensitive process and requires pregnant women to have all the knowledge needed for a healthy outcome for both mother and child. Varner (2015) stated that childbirth educators are a group of educators who deliver proper education, support, and preparation to women before, during, and after childbirth. 
Danawi et al. (2016) reported that teenage pregnancy is associated with a low level of education and poverty, making it difficult to delay pregnancy. Early exposure to sexual intercourse and repeated childbirth experiences exposes women to infection with HPV serotype 16 and 18 that may develop into cervical cancer (Asamu, 2018). HPV is a double-stranded DNA that infects the cutaneous epithelia and mucosal surfaces of female cervix (Chiantore et al., 2020). Childbirth educators have the responsibility to educate women about early screening for HPV and other related sexually transmitted diseases. Inadequate screening adherence results in the development of cervical cancer (Larkey et al., 2012).

\section{Pregnant Women and Health Education}

Pregnant women are denied access to health education based on their socioeconomic, cultural, and educational level (Silva et al., 2020). Strayer et al. (2020) reported that health information and implementation are thwarted before reaching the community members, preventing pregnant women from understanding availability of childbirth educators. Strayer et al. argued that, in many communities, there is a lack of communication and interpretation of health services available to the community members.

Cullerton et al. (2016) argued that low participation in cervical cancer screening is due to lack of education on attitudes, intentions, and knowledge among culturally diverse communities. Martens et al. (2016) noted that Hispanics in the United States may have a better understanding of early screening for cervical cancer but lack access to healthcare resources, and language barriers result in lack of screening or inadequate screening.

A study by Greenfield et al. (2019) found that pregnant women faced concerns regarding childbirth. Pregnant women searched for information about choices they can make during pregnancy (i.e., cesarean section vs. vaginal birth) and analyzed their health risks after birth (Greenfield et al., 2019). Suto et al. (2017) reported that women given prenatal education by childbirth educators received information about the labor and delivery processes and the care of their babies and themselves after birth.

\section{Pregnancy and Cervical Cancer}

Cervical cancer caused by HPV is one of the gynecological malignancies that most complicates pregnancy, but occurrence during pregnancy is preventable, and treatment is based on stages of cervical cancer (Perrone et al., 2019). Beharee et al. (2019) reported an increased incidence of $71.6 \%$ in cervical cancer due to increased incidence of pregnancy among older women, missed diagnosis, and limited examinations. Pregnant women, therefore, need more information to decide on treatment strategies when they are diagnosed with cervical cancer in pregnancy and more information for future prevention of the disease (Perrone et al., 2019).

\section{Purpose of the Study}

The purpose of our study was to identify barriers affecting childbirth educators in offering health education to pregnant women to reduce cervical cancer. We aimed to examine the risk factors associated with pregnancy and cervical cancer and the role played by childbirth educators in disseminating information to pregnant women to prevent the development of cervical cancer.

\section{Research Questions}

The following research questions guided this research study:

Research Question 1: Is women's lack of health education by childbirth educators a barrier to survival from cervical cancer?

Research Question 2: Is women's lack of access to childbirth educators a barrier to survival from cervical cancer in the United States? 
Research Question 3: Is lack of availability of childbirth educators in the United States a barrier to survival from cervical cancer in the United States?

\section{Methods}

This was a secondary analysis of data obtained from the Surveillance Epidemiology and End Results (SEER) between 1973 and 2012. The Statistical Package for Social Science (SPSS) was used to analyze the data. Descriptive statistics and logistic regression analysis were used to describe the sample and to determine the association among the variables.

\section{Sampling}

We employed two sampling procedures: a random sampling from the data population, and a purposive sampling of childbirth educators in the United States. We employed OpenEpi for sample size calculation, power $(1$-beta $)=80 \%$, the odds ratio $=2$, and the confidence interval $(C I)=95 \%$. The sample size through the Fleiss method was 1,425 . However, we used all cases in the data that met the inclusion criteria.

\section{Inclusion and Exclusion Criteria}

All men were excluded from the study. Women with other malignancies that are not cervical cancer were excluded. Women 20 years of age and older at the time of diagnosis with cervical cancer, whether pregnant or not, were included. We obtained IRB approval to use and analyze the SEER data for our study.

\section{Results}

There were 520,153 cases included in this study; 27.1\% $(n=140936)$ were women between 20 and 50 years of age, while $72.9 \%(n=379217)$ were women greater than 50 years old (Table 1$)$.

Table 1. Demonstrated Characteristics of the Sample

\begin{tabular}{lll}
\hline Variable & Frequency & Percentage (\%) \\
\hline Females & 520,153 & 100 \\
\hline Age: & & \\
$20-29$ & 16,725 & $3 \cdot 2$ \\
$30-39$ & 41,594 & 8 \\
$40-49$ & 82,617 & $15 \cdot 9$ \\
$>50$ & 379,217 & 72.9 \\
Total $N:$ & 520,153 & 100 \\
\hline Race: & & \\
White & 448,739 & 86.3 \\
Nonwhite & 66,918 & 12.9 \\
Missing & 4496 & 0.9 \\
Total N: & 520,153 & 100 \\
\hline U.S. regions: & & \\
Alaska & 499 & 0.1 \\
East & 173,932 & $33 \cdot 4$ \\
Northern Plains & 76,990 & 14.8 \\
Southwest & 32,595 & 6.3 \\
Pacific Coast & 236,137 & $45 \cdot 4$ \\
Total N: & 520,153 & 100 \\
\hline
\end{tabular}


Table 2. Availability of Childbirth Educators per U.S. Regions

\begin{tabular}{ll}
\hline U.S. regions & $\begin{array}{l}\text { Average number of childbirth } \\
\text { educators per region }\end{array}$ \\
\hline Alaska & 630 \\
East & 227 \\
Northern Plains & 512 \\
Southwest & 529 \\
Pacific Coast & 495 \\
\hline
\end{tabular}

\section{Result of Research Question 1}

Is lack of health education by childbirth educators a barrier to survival from cervical cancer?

Lack of health education was a statistically significant barrier to surviving cervical cancer. Of the pregnant women, $36 \%$ had access to childbirth education in early pregnancy. Early health education by childbirth educators led to a reduced incidence of cervical cancer, and the odds of dying were reduced to .917, while late education by childbirth educators increased the odds of dying by 1.0905. These results indicate that lack of early health education is a barrier to survival from cervical cancer. The binary logistic regression showed that health education was statistically significant $\left(\chi^{2}[4]=52.685, p=0.000\right)$. Health education was a significant predictor of cervical cancer survival.

\section{Results of Research Question 2}

Is women's lack of access to childbirth educators a barrier to survival from cervical cancer in the United States?

Access to childbirth educators was a statistically significant barrier to surviving cervical cancer. The results of binary logistic regression indicated that access to childbirth educators was a statistically significant predictor $\left(\chi^{2}[4]=550.120, p=0.000\right)$ of survival from cervical cancer.

\section{Results of Research Question 3}

Is lack of availability of childbirth educators a barrier to survival from cervical cancer in the United States?

We used binary logistic regression analysis to study the association between five regions in the United States for availability of childbirth educators and the survival of pregnant women who received health education by childbirth educators. Geographic region was a statistically significant predictor $\left(\chi^{2}[4]=146.286, p<.005\right)$ of survival from cervical cancer. Regions with more childbirth educators demonstrated an increased access by pregnant women to childbirth education.

Alaska had more childbirth educators compared to the other regions. The odds of dying were lower among women in Alaska exposed to health education by childbirth educators; it had decreased odds ratio of dying by .714 compared to women on the Pacific Coast with a 1.009 odds ratio of dying. The women in the Southwest had reduced odds ratio of dying of .918 from cervical cancer compared to the women in the Pacific Coast region. The women in the Eastern United States who received late health education by childbirth educators had an increased likelihood of dying by 1.044 times compared to the women in the Pacific Coast region. The women living in the Northern Plains had .997 reduced odds of dying compared to the women in the Pacific Coast region. The women who resided in the Southwest also had a decreased likelihood of dying with an odds ratio of .918 compared to the women in the Pacific Coast region. There was no statistically significant 
difference in the odds of dying from cervical cancer between women in the Northern Plains and women on the Pacific Coast $(p=.718)$.

\section{Discussion}

In our study, women with late childbirth education accounted for $68.6 \%$ of pregnant women in the labor room. In some regions of the United States, childbirth educators were not evenly distributed. Access to healthcare was a statistically significant barrier in surviving cervical cancer. Our study showed that $36 \%$ of pregnant women had access to childbirth educators, and $64 \%$ of pregnant women passed through the delivery processes without being properly educated about their healthcare and cervical cancer prevention.

Our study showed that women in some regions of the United States received unequal access to health education that led to increased death from cervical cancer. Women from the East and Northern Plains have higher odds of dying from cervical cancer than women in Alaska and the Pacific Coast due to differences in early childbirth educators' intervention.

\section{Significance of the Study}

Pregnant women benefited from health education by qualified childbirth educators. Lack of health education for pregnant women was a barrier to cervical cancer survival. Research Question 1 revealed that early health education for pregnant women by childbirth educators reduced the odds of dying from cervical cancer.

\section{Interpretation of the Findings}

Childbirth educators are helpful in the dissemination of information to pregnant women regarding health needs during pregnancy. Moreover, they are not only helpful to pregnant women but also to their partners who assist during pregnancy, birth, and thereafter. Childbirth educators advise women on immunization during pregnancy and prevention of cervical cancer by early screening for HPV or Pap smear. Our study revealed that early education by childbirth educators improved survival from cervical cancer when pregnant women got screened for HPV early in life. Our study reinforced that lack of access to childbirth educators reduces health information dissemination to pregnant women, results in fewer pregnant women who get screened, and reduces survival from cervical cancer deaths.

\section{Limitations of the Study}

This is a cross-sectional quantitative study. We used the secondary data from SEER between 1973 and 2012 to examine the pregnant women who received early health education from childbirth educators and survived cervical cancer and those pregnant women who did not receive early health education from childbirth educators and their outcomes from cervical cancer. Our study was limited to pregnant women living in the United States and may not be generalizable to pregnant women living outside the United States. Our study did not identify the treatment modality of pregnant women during the pregnancy and the HPV types that led to the development of cervical cancer.

\section{Recommendations for Future Research}

Globally, cervical cancer is one of the deadly diseases, and early health education for pregnant women by health educators must be encouraged in all hospitals. Childbirth educators must be trained to give health education to pregnant women about causes, immunizations, and screening for cervical cancer. Pregnant women must be given access to healthcare centers and access to childbirth educators for guidance through 
childbirth. Young adults should be encouraged to study childbirth education as a profession to increase the availability of the childbirth educators in all hospitals.

\section{Implications of the Findings}

The outcome of our study offers hope for the reduction of cervical cancer through health education for pregnant women. Our study is a resource for policy makers to formulate strategies that will enable childbirth educators to (1) have legal status to educate pregnant women and (2) grant them easy access to healthcare and procedures for Pap smear screening and HPV immunizations to prevent the development of cervical cancer. Our study encouraged pregnant women to undergo screening for the HPV virus and encouraged their children (both boys and girls) to obtain the HPV vaccine. The outcome is reduced infection by HPV and deaths from cervical cancer.

\section{Summary and Conclusions}

The results of our study revealed that pregnant women who received early childbirth education avoided cervical cancer, while pregnant women who received late education were at greater risk for cervical cancer later in life. The access to childbirth educators and the availability of childbirth educators were barriers to acquiring knowledge to make sound health decisions related to prevention of cervical cancer. Our study revealed the contribution of childbirth educators in delivering health education to pregnant women. This education helped women remain healthy during pregnancy and to detect and survive cervical cancer. To support pregnant women, policy makers must ensure that pregnant women have access to childbirth education. 


\section{References}

Asamu, O. O. (2018). Early radiation therapy and cervical cancer survival in the United States [Doctoral dissertation, Walden University].

Beharee, N., Shi, Z., Wu, D., \& Wang, J. (2019). Diagnosis and treatment of cervical cancer in pregnant women. Cancer Medicine, 8(12), 5425-5430. https://doi.org/10.1002/cam4.2435

Chiantore, M. V., Mangino, G., Iuliano, M., Capriotti, L., Di Bonito, P., Fiorucci, G., \& Romeo, G. (2020). Human papillomavirus and carcinogenesis: Novel mechanisms of cell communication involving extracellular vesicles. Cytokine \& Growth Factor Reviews, 51, 92-98. https://doi.org/10.1016/j.cytogfr.2019.12.009

Cullerton, K., Gallegos, D., Ashley, E., Do, H., Voloschenko, A., Fleming, M., Ramsey, R., \& Gould, T. (2016). Cancer screening education: Can it change knowledge and attitudes among culturally and linguistically diverse communities in Queensland, Australia? Health Promotion Journal of Australia, 27(2), 140-147. https://doi.org/10.1071/HE15116

Cutajar, L., Miu, M., Fleet, J.-A., Cyna, A., \& Steen, M. (2020). Antenatal education for childbirth: Labour and birth. European Journal of Midwifery, 4. https://doi.org/10.18332/ejm/120002

Danawi, H., Bryant, Z., \& Hasbini, T. (2016). Targeting unintended teen pregnancy in the U.S. International Journal of Childbirth Education, 31(1), 28-31.

Davis, D., Ferguson, S., Nissen, J., Fowler, C., \& Mosslar, S. (2019). A salutary childbirth education program: Health promoting by design. A discussion paper. Sexual \& Reproductive Healthcare, 22, 100456. https://doi.org/10.1016/j.srhc.2019.100456

Deeb-Sossa, N., \& Kane, H. (2017). Pregnancy without women: Lessons from childbirth classes. Sexuality Research and Social Policy, 14(4), 380-392. https://doi.org/10.1007/s13178-016-0265-6

Furry, D. B., Mashalla, Y., \& Tshweneagae, G. T. (2019). Sexual and reproductive health among high school adolescents in West Shoa zone, Oromia region in Ethiopia. African Journal of Reproductive Health, 23(1), 65-72. https://doi.org/10.29063/ajrh2019/v23i1.7

Gower, S., Van den Akker, J., Jones, M., Dantas, J. A. R., \& Duggan, R. (2016). Australian nursing and midwifery educators delivering evidence-based education in Tanzania: A qualitative study. Nurse Education in Practice, 18, 16-22. https://doi.org/10.1016/j.nepr.2016.02.009

Greenfield, M., Jomeen, J., \& Glover, L. (2019). “It can't be like last time"-Choices made in early pregnancy by women who have previously experienced a traumatic birth. Frontiers in Psychology, 10, 56. https://doi.org/10.3389/fpsyg.2019.00056

Kervella, L., Berveiller, P., Bourdillon, M., \& Rousseau, A. (2020). Midwives' practices related to cervical cancer screening during pregnancy: A vignette-based study. Sexual \& Reproductive Healthcare, 26, 100539. https://doi.org/10.1016/j.srhc.2020.100539

Larkey, L. K., Herman, P. M., Roe, D. J., Garcia, F., Lopez, A. M., Gonzalez, J., Perera, P. N., \& Saboda, K. (2012). A cancer screening intervention for underserved Latina women by lay educators. Journal of Women's Health, 21(5), 557-566. https://doi.org/10.1089/jwh.2011.3087

Luce, A., Cash, M., Hundley, V., Cheyne, H., van Teijlingen, E., \& Angell, C. (2016). “Is it realistic?” The portrayal of pregnancy and childbirth in the media. BMC Pregnancy and Childbirth, 16(1), 40. https://doi.org/10.1186/s12884-016-0827-x

Mamo, L., \& Epstein, S. (2017). The new sexual politics of cancer: Oncoviruses, disease prevention, and sexual health promotion. BioSocieties, 12(3), 367-391. https://doi.org/10.1057/biosoc.2016.10 
Martens, C. E., Crutchfield, T. M., Laping, J. L., Perreras, L., Reuland, D. S., Cubillos, L., Pignone, M. P., \& Wheeler, S. B. (2016). Why wait until our community gets cancer? Exploring CRC screening barriers and facilitators in the Spanish-speaking community in North Carolina. Journal of Cancer Education, 31(4), 652-659. https://doi.org/10.1007/s13187-015-0890-4

Perrone, A. M., Bovicelli, A., D’Andrilli, G., Borghese, G., Giordano, A., \& De Iaco, P. (2019). Cervical cancer in pregnancy: Analysis of the literature and innovative approaches. Journal of Cellular Physiology, 234(9), 14975-14990. https://doi.org/10.1002/jcp.28340

Radecki Breitkopf, C., Finney Rutten, L. J., Findley, V., Jacobson, D. J., Wilson, P. M., Albertie, M., Jacobson, R. M., \& Colón-Otero, G. (2016). Awareness and knowledge of human papillomavirus (HPV), HPVrelated cancers, and HPV vaccines in an uninsured adult clinic population. Cancer Medicine, 5(11), 3346-3352. http://doi.org/10.1002/cam4.933

Sherris, J., Herdman, C., \& Elias, C. (2001). Beyond our borders: Cervical cancer in the developing world. Western Journal of Medicine, 175(4), 231-233. https://doi.org/10.1136/ewjm.175.4.231

Silva, C. C. D., Savian, C. M., Prevedello, B. P., Zamberlan, C., Dalpian, D. M., \& Santos, B. Z. D. (2020). Access and use of dental services by pregnant women: An integrative literature review. Ciencia \& Saude Coletiva, 25, 827-835. https://doi.org/10.1590/1413-81232020253.01192018

Strayer III, T. E., Kennedy, L. E., Balis, L. E., Ramalingam, N. S., Wilson, M. L., \& Harden, S. M. (2020). Cooperative extension gets moving, but how? Exploration of extension health educators' sources and channels for information-seeking practices. American Journal of Health Promotion, 34(2), 198-205. https://doi.org/10.1177/0890117119879606

Suto, M., Takehara, K., Yamane, Y., \& Ota, E. (2017). Effects of prenatal childbirth education for partners of pregnant women on paternal postnatal mental health and couple relationship: A systematic review. Journal of Affective Disorders, 21O, 115-121. https://doi.org/10.1016/j.jad.2016.12.025

Suzuki, S., Hayata, E., Hoshi, S. I., Sekizawa, A., Sagara, Y., Tanaka, M., Kinoshita, K., \& Kitamura, T. (2021). Current status of cervical cytology during pregnancy in Japan. Plos One, 16(1), e0245282. https://doi.org/10.1371/journal.pone.0245282

Varner, C. A. (2015). Comparison of the Bradley method and HypnoBirthing childbirth education classes. The Journal of Perinatal Education, 24(2), 128-136. https://doi.org/10.1891/1946-6560.24.2.128

Waller-Wise, R. (2011). Umbilical cord blood: Information for childbirth educators. The Journal of Perinatal Education, 2O(1), 54-6o. https://doi.org/10.1891/1058-1243.20.1.54

Zauderer, C. (2009). Postpartum depression: How childbirth educators can help break the silence. The Journal of Perinatal Education, 18(2), 23-31. https://doi.org/10.1624/105812409X426305

Zhang, S., Xu, H., Zhang, L., \& Qiao, Y. (2020). Cervical cancer: Epidemiology, risk factors and screening. Chinese Journal of Cancer Research, 32(6), 720-728.

https://doi.org/10.21147/j.issn.1000-9604.2020.06.05

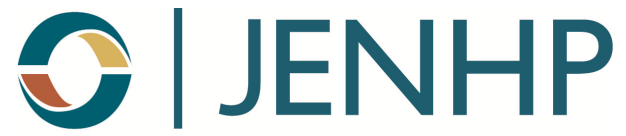

The Journal of Excellence in Nursing and Healthcare Practice welcomes manuscripts focusing on topics such as national and international workforce issues, quality improvement projects, evidence-based practice initiatives, nursing research studies, interprofessional practice, educational issues, telehealth, improvements in technology, and the impact of social change in society. 